ISSN electrónico: $1585-5210$

DOI: https://doi.org/10.14201/rmc.27907

\title{
HUMANIZACIÓN DE LA MEDICINA EN TIEMPOS DE COVID-19
}

\section{Humanization of medicine in times of COVID-19}

\author{
Agustín HIDALGO BALSERA
}

Área de Farmacología, Departamento de Medicina, Facultad de Medicina y Ciencias de la Salud, Universidad de Oviedo. Instituto Universitario de Oncología del Principado de Asturias (IUOPA), Fundación Cajastur. Instituto de Investigación Sanitaria de Asturias (ISPA) (España).

Correo electrónico: hidalgo@uniovi.es

Recibido: 11 de agosto de 2021

Aceptado: 17 de agosto de 2021

"Donde quiera que se ama el arte de la medicina se ama también a la humanidad»

(Platón)

La peor pandemia del siglo XXI ha puesto sobre la mesa problemas de enorme calado científico, social y humanístico. Es evidente que la pandemia sanitaria ha tenido y tiene profundas repercusiones económicas, productivas y de organización y relación social; pero también lo es que ha comprometido la relación entre médicos y enfermos, al menos en la Atención Primaria, derivada a una prestación sanitaria telefónica más impersonal, más distante $y$, tentativamente, menos cálida y empática porque, entre otras cosas, ni el médico ni el enfermo pueden leer las expresiones faciales del otro ni pueden sentir el calor del tacto. Lo que, bien entendido, es un déficit tanto de relación humana como de humanismo médico.
Esta situación se ha trasladado a las aulas y ha modificado profundamente las tecnologías docentes dejándolas en buena medida en la docencia telemática en la que teníamos poca experiencia, hemos tenido que aprender deprisa y asumir que ha llegado para quedarse ${ }^{1}$. En esta tesitura, diversos autores, profesores y estudiantes, se han formulado preguntas y evaluado resultados. Así, Millán² se pregunta "¿Se puede enseñar medicina sin enfermos? ¿Se puede enseñar medicina a distancia?" Lo que no deja de ser complejo porque por una parte "El paciente nunca es igual a lo que ponen los libros; los padecimientos de un enfermo no siempre son lo mismo que las enfermedades», $y$, por otra, «En 
la enseñanza en un entorno clínico se adquieren valores propios de la profesión. Entrega, generosidad, altruismo, trabajo en equipo, compasión, sufrimiento, comunicación, etc. No en vano se ha propuesto que "No hay ninguna época mejor para aprenderlo que una pandemia ${ }^{3} »$.

Sin embargo, la no presencialidad de la docencia ha condicionado que "Los estudiantes han perdido la oportunidad de cimentar su decisión vocacional de dedicarse a la medicina. También han perdido la oportunidad de enfrentar problemas éticos complejos, como actuar cuando los recursos son limitados y a gestionar la incertidumbre ${ }^{2}$ ». Y, cabría añadir, la virtualización de la enseñanza impide el contacto con el enfermo y, en consecuencia, rompe dos diálogos imprescindibles en medicina: la relación entre el profesor y el estudiante y entre el estudiante y el enfermo supervisada por sus tutores, de quienes aprenderán toda una suerte de conductas y valores médicos imprescindibles para su formación, entre ellos, la aproximación al enfermo, la apreciación del diálogo médico - enfermo adecuada no sólo al tipo y gravedad de la enfermedad sino también a su formación cultural y rol social, porque la medicina no puede dejar de ser social $y$, por tanto humana ${ }^{4}$.

Por otra parte, se han propuesto una serie de cambios que deberían implementarse para afrontar la nueva situación, con especial referencia a los nuevos contenidos que deberemos impartir, a las metodologías docentes que deberemos usar y el papel de las TIC, la adaptación de las evaluaciones a la nueva situación, y a los nuevos roles que deberán desempeñar tanto profesores como estudiantes que viven la situación actual con ansiedad e incertidumbre ${ }^{5}$. Y parece claro que La COVID-19 ha evidenciado algunas necesidades no cubiertas del ejercicio profesional, entre ellas que se requieren médicos con competencias generales capaces de desenvolverse en la clínica, que estén instruidos en la resolución de conflictos bioéticos diariamente y con habilidades de comunicación ${ }^{6}$, competencias trasversales recogidas en los planes de estudio de los Grados en Medicina, a cuyo desarrollo contribuye la Revista de Medicina y Cine.

En cuanto a los alumnos, un estudio comparativo con grupos de expuestos a enseñanza presencial y telemática sugieren que los estudiantes «prefieren la educación presencial sobre todo por el contacto con los compañeros y los profesores ${ }^{7} »$. La evaluación realizada por asociaciones de estudiantes de la Universidad de Murcia sugiere que la enseñanza telemática aumentó la carga de trabajo percibida y una gran variabilidad en las modalidades usadas para suplir la docencia presencial ${ }^{8}$. Una evaluación en la universidad Francisco de Vitoria, resalta que los estudiantes encuentran como aspectos favorables la autoevaluación y el sistema de gestión de dudas, mientras que el profesorado ensalza la autonomía del estudiante y la necesidad adicional de presencialidad. Se considera negativa la falta de prácticas y otras características propias de la enseñanza presencial o la tutoría personalizada, entre otros déficits 9 .

En la situación clínica extrema generada por la COVID-19, en la que el enfermo está sólo ante la enfermedad y sus dificultades, huérfano de toda ayuda familiar, el apoyo humano del personal sanitario ha sido esencial, razón por la que debemos profundizar en esta formación humana y humanística de nuestros estudiantes de medicina y en los de otras ciencias de la Salud. Por eso resulta sorprendente que no se haga alusión alguna en los textos reseñados a la necesidad de profundizar en el humanismo médico sobre todo si tenemos en cuenta la escasa oferta de materias en los grados de medicina ya que se centra preferentemente en historia de la medicina y en bioética, pero hay déficits importantes en otras como sociología, artes visuales, cine, antropología o literatura, son mejorables las estrategias de impartición ${ }^{10} y$, por la escasez con que se practica, tenemos poca experiencia en la enseñanza de valores.

También ha quedado claro que, como se recoge en la Declaración Complutense ${ }^{11}$, la 
medicina tiene como objetivo el enfermo y no la enfermedad, y que, precisamente por ello, «el encuentro con el enfermo y el encuentro con el alumno al lado del enfermo, son partes esenciales para introducir al alumno en el universo de una medicina humanizada", lo que obliga a la promoción del «desarrollo humano» de los estudiantes a lo largo del curriculum formativo, para lo que es importante la existencia de unidades docentes ${ }^{12}$ en los centros educativos que guíen el desarrollo curricular de los estudiantes en los aspectos humanos y humanísticos porque estimulan la sensibilidad médica y los convertirá en mejores médicos, en médicos de enfermos y no de enfermedades.

No es sólo la medicina científica la que aplica a la COVID-19 sino también la que se practica con el corazón $n^{13}$. La cercanía del médico está más allá de la ciencia. Cada acto médico implica toda una historia de la medicina y de la ciencia pasada por el cedazo de los logros sociales, las disciplinas humanas, los principios éticos, y el respeto de la actitud del enfermo ante la enfermedad. $Y$ esto requiere profundizar en el perfil formativo en humanidades médicas para conseguir esos médicos de enfermos que, cada vez con más insistencia, demanda la sociedad. A ello viene contribuyendo durante 16 años, con una atracción creciente entre sus lectores, la Revista de Medicina y Cine.

\section{Referencias}

1. Pérez-Sayáns $M$, Chamorro CM, Reboiras $D$, Gándara P, Gallas P. Percepción por los docentes de la Facultad de Medicina y Odontología de la USC sobre la docencia virtual y sincrónica tras la crisis delCovid-1. Rev. Esp. Edu. Med. 2020;2: 53-64.

2. Millán Núñez-Cortés J. Influencia de la pandemia por COVID-19 en la formación médica. Rev. Med. Cine. 2020; $16(\mathrm{e}): 25-31$.
3. Lucey $C R$, Johnston SC. The transformational effects of COVID-19 on Medical Education. JAMA. 2020;324(11):1033-1034.

4. Trainini JC. Humanización en la práctica médica. Educ. Med. 2021; 21(2):65-66.

5. Palés-Argullós J; Gomar-Sancho C. La educación médica en el grado en medicina en época de pandemia y de post-pandemia covid-19. Rev. Med. Cine. 2020; $16(\mathrm{e}): 13-23$.

6. Millán Núñez-Cortés J. Con o sin pandemia, el paciente siempre en el foco de la educación médica. Educ. Med. 2021;22(1):1.

7. Bru S, Ribeiro MPC, Quandt E, Clotet J, Jiménez J. Covid-19, an opportunity to compare in-person and online teaching. Rev. Esp. Edu. Med. 2021;2:72-83.

8. Rojo Martínez JM, Conesa Erragbaoui AG, Vera Villalba J, García García MA, Martínez de Rituerto Rey P, Cano Dólera B et al. Encuesta sobre docencia online en las Facultades de Enfermería y Medicina de la Universidad de Murcia durante COVID-19. Rev. Esp. Edu. Med 2020;1(1):24-31.

9. Ruiz Moral R, Sierra Isturiz J, García-Miguel MJ, Cerro A, García de Leonardo C, Turpín Sevilla MC, et al. Opiniones de profesores y alumnos sobre un programa integral online en medicina durante el confinamiento por COVID-19. Educ. Med. 2021; 22(4): 206-214.

10. Orefice C, Pérez J, Baños JE. The presence of humanities in the curricula of medical students in Italy and Spain. Educ. Med. 2019; 20(Suppl 1):79-82.

11. Arias J. Declaración complutense. Decálogo de las facultades de medicina de Madrid: en pro de una medicina centrada en el paciente. Educ. Med. 2021; 22(1):40-41.

12. Palés-Argullós J, Nolla-Domenjó M. Competencias transversales, un tema pendiente en las facultades de medicina. FEM 2016; 19(5):227-228.

13. Sacristán JA, Milán J. El médico frente a la COVID19: lecciones de una pandemia. Educ. Med. 2020; 21(4):265-271. 


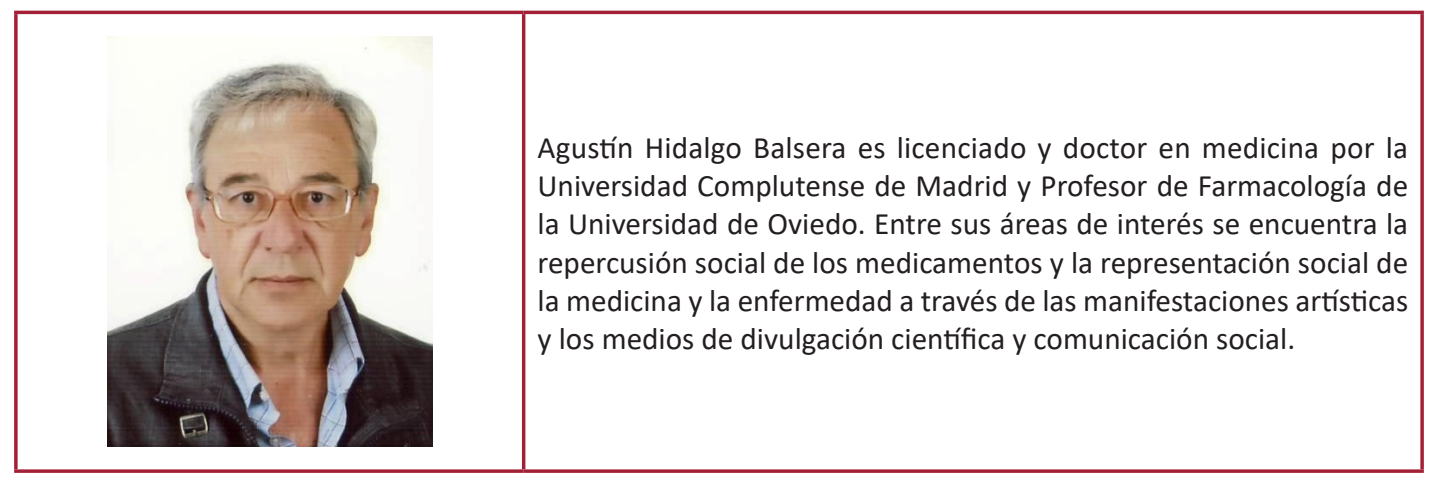

